\title{
SYMMETRY PROPERTIES OF THE SOLUTIONS TO THOMAS-FERMI-DIRAC-VON WEIZSÄCKER TYPE EQUATIONS
}

\author{
RAFAEL D. BENGURIA AND CECILIA YARUR
}

\begin{abstract}
We consider a semilinear elliptic equation with a spherically symmetric potential (specifically, Thomas-Fermi-Dirac-von Weizsäcker type equations without electronic repulsion). Assuming some regularity properties of the solutions at the origin and at infinity, we prove that the solutions have spherical symmetry.
\end{abstract}

\section{INTRODUCTION}

In this article we shall discuss symmetry properties of the solutions $u(x)$ of the problem

$$
-\Delta u-Z u /|x|+a(u)=0 \quad \text { in } \mathbb{R}^{N}, \quad(N \geq 2),
$$

such that $u(x) \rightarrow 0$ as $|x| \rightarrow \infty$. In (1), $Z$ is a positive real constant and the function $a(t)$ is locally Lipschitz continuous for $t \in \mathbb{R}$, and $a(0)=0$.

Problem (1) (for $N=3$ ) arises in the Thomas-Fermi-von Weizsäcker theory of atoms and molecules $[3,10]$ without electronic repulsion. There, $Z /|x|$ is the electric potential due to a fixed nucleus of atomic number $Z$ located at the origin. $u(x)^{2}$ stands for the electronic density and $\int u(x)^{2} d x$ is the total number of electrons.

Here we prove two different types of results about the solutions of problem (1). The first type concerns the spherical symmetry of the solutions to (1) and is embodied in the following two theorems:

Theorem 1. Let $Z>0$, and $\lambda \geq(Z /(N+1))^{2}$. Let $u$ be a solution of

$$
-\Delta u-Z u /|x|+a(u)=0 \quad \text { in } \mathbb{R}^{N} \backslash\{0\} .
$$

Suppose that $g(u)=a(u)-\lambda u$ is a nondecreasing function of $u$ for $\lambda>$ $(Z /(N+1))^{2}$, and strictly increasing if $\lambda=(Z /(N+1))^{2}$. If $u$ satisfies the

Received by the editors October 14, 1988.

1980 Mathematics Subject Classification (1985 Revision). Primary 35J60; Secondary 35A05, 35Q20, 82A99.

Key words and phrases. Semilinear elliptic equations, Thomas-Fermi-Dirac-von Weizsäcker equations, uniqueness of positive solutions, spherical symmetry of solutions.

This work was partially supported by FONDECYT grant 132-88. The first author was also supported by Departamento Técnico de Investigación, Universidad de Chile (E-2852-8812). The second author was also supported by DICYT (Universidad de Santiago) grant 04-8533-YS. 
following two conditions:

(i) $\lim _{r \rightarrow 0} r^{N-1}\|u-\bar{u}\|_{L^{2}\left(S^{N-1}\right)}=0$, and

(ii) $\lim _{r \rightarrow \infty} r^{N-1-K} \exp \left(-r \lambda^{1 / 2}\right)\|u-\bar{u}\|_{L^{2}\left(S^{N-1}\right)}=0$, with $K=\frac{1}{2}\left((N-1)-Z \lambda^{-1 / 2}\right)$,

then $u \equiv \bar{u}$, where $\bar{u}$ denotes the spherical average of $u$, i.e.

$$
\bar{u}(r) \equiv \int_{S^{N-1}} u(r \sigma) d \sigma .
$$

Remark. If $\lambda=(Z /(N+1))^{2}$ and $a(u)=(Z /(N+1))^{2} u$, the theorem does not hold. In fact, $-(Z /(N+1))^{2}$ is the second eigenvalue of $-\Delta-Z /|x|$ in $\mathbb{R}^{N}$.

Theorem 2. Assume that $a(s) / s$ is nondecreasing in $(0, \infty)$ and let $u$ be a positive solution of

$$
-\Delta u-Z u /|x|+a(u)=0 \quad \text { in } \mathbb{R}^{N} \quad(N \geq 2)
$$

such that $u(x) \rightarrow 0$ as $|x| \rightarrow \infty$. Then $u=\bar{u}$.

Remark. In [11], Lieb proved a theorem (Theorem 5, p. 20) very similar to this theorem. Moreover, Lieb's paper (see also [12]) gave the first (and complete) treatment of the Thomas-Fermi-von Weizsäcker equation without electronic repulsion. The theorems differ in that Lieb takes a specific form for $a(s)$ (namely $\left.a(s)=|s|^{4 / 3} s\right)$ whereas we assume only that $a(s) / s$ is nondecreasing and Lieb assumes $u \in L^{6}$ whereas we assume that $u$ goes to 0 at infinity. Furthermore, the two methods of proof are quite dissimilar.

The second type of results concerns the nonexistence of nontrivial solutions of problem (1) under certain conditions on the nonlinear term (i.e. on $a(\cdot)$ ) . These are summarized in the following.

Theorem 3. Let $\lambda>(Z /(N-1))^{2}, Z>0$, and $(a(u)-\lambda u) \operatorname{sgn}(u) \geq 0$. Then, if. $u$ is a solution of (2) (in the sense of distributions) such that

(i) $\lim _{r \rightarrow 0} r^{N-2} \int_{S^{N-1}}|u(r \sigma)| d \sigma=0$, for $N \geq 3$, or

(i' ${ }^{\prime} \lim _{r \rightarrow 0}|\log (r)|^{-1} \int_{S^{1}}|u(r \sigma)| d \sigma=0$, for $N=2$, and

(ii) $\lim _{r \rightarrow \infty} \exp \left(-r \lambda^{1 / 2}\right) r^{-K+N-1} \int_{S^{N-1}}|u(r \sigma)| d \sigma=0$, for $N \geq 2$,

where $K=\frac{1}{2}\left((N-1)-Z \lambda^{-1 / 2}\right)$, we have $u \equiv 0$.

The rest of the paper is organized as follows. In $\S 2$ we prove two lemmas about second order ordinary differential equations, which are needed in the sequel. In $\S 3$ we prove Theorems 1 and 2 . Finally, in $\S 4$ we prove the nonexistence results.

\section{Preliminary Lemmas}

In this section we prove some existence lemmas concerning second order ordinary differential equations, which are needed in the proof of the main results of this paper. 
Lemma 4. Let $Z>0$ and $\lambda>(Z /(N+1))^{2}$. Then, there exists a solution of (3) $h^{\prime \prime}(r)+\frac{N-1}{r} h^{\prime}(r)-\frac{N-1}{r^{2}} h(r)+\frac{Z}{r} h(r)-\lambda h(r)=0, \quad r \in(0,+\infty)$, such that

(i) $h>0$ in $(0, \infty)$,

(ii) $\lim _{r \rightarrow \infty} \exp \left(r \lambda^{1 / 2}\right) r^{K} h(r)=1$, where $K=\frac{1}{2}\left((N-1)-Z \lambda^{-1 / 2}\right)$ and $\lim _{r+\infty} h^{\prime}(r) / h(r)=-\lambda^{1 / 2}$, and

(iii) $\lim _{r \rightarrow 0} r^{N-1} h(r)=C(N, Z, \lambda)$, where $C$ is a positive constant.

Remark 1. For $\lambda=(Z /(N+1))^{2}, h(r) \equiv r \exp (-r Z /(N+1))$ is a solution of (1) satisfying conditions (i), (ii) above but not condition (iii).

Remark 2. If $(a(u)-\lambda u) \operatorname{sgn}(u) \geq 0$ and $\lambda>(Z /(N-1))^{2}$, we shall see (proof of Theorem 3 below) that the only solution of

$$
\Delta u+Z u /|x|=a(u) \quad \text { in } \mathbb{R}^{N} \quad(N \geq 2),
$$

such that $u(x) \rightarrow 0$ when $|x| \rightarrow \infty$ is the trivial solution $u \equiv 0$. Thus, for the purpose of this article we would only need to consider the case $\lambda \leq$ $(Z /(N-1))^{2}$ in Lemma 4 . However, we give the general proof of this lemma for completeness.

Proof. Let us consider $w(r)=r^{-K} \exp \left(-r \lambda^{1 / 2}\right)$, and let $g$ be defined by

$$
h(r)=w(r) g(t),
$$

where $t^{\prime}(r) \equiv d t / d r=r^{1-N} / w(r)^{2}$. Then, $g(t)$ satisfies

$$
\ddot{g}(t)=d^{2} g / d t^{2}=X(t) g(t),
$$

where $X(t) \equiv(1+K)(N-1-K) /\left(r t^{\prime}(r)\right)^{2}$. From the definition of $K$ it follows that $N-1-K>0$. Moreover, since $\lambda>(Z /(N+1))^{2},(1+K)>0$. Thus, $X(t)>0$. Now, we consider the cases $K+(2-N) / 2>0$ and $K+(2-N) / 2 \leq 0$ separately.

Case 1. $(K+(2-N) / 2)>0$. Here $t(r)=\int_{0}^{r}\left(s^{1-N} / w(s)^{2}\right) d s<\infty$ and $t \in(0,+\infty)$ for $r \in(0,+\infty)$.

Case 2. $(K+(2-N) / 2) \leq 0$. Here $t \in(-\infty,+\infty)$ for $r \in(0,+\infty)$.

We shall show that

$$
\int^{\infty} t X(t) d t<\infty
$$

in both cases. Once (6) is proven, it follows from [7, Theorem 9.1, Chapter XI] that there exists a solution $g(t)$ of (5) such that (a) $\lim _{t \rightarrow+\infty} g(t)=1$, and (b) $\dot{g}(t)=o(1 / t)$ as $t \rightarrow+\infty$. Then, from (a), (b) and Definition (4), (ii) follows. Now, since $X(t)>0$, the solutions of (4) vanish at most once on the interval $\left(t_{0},+\infty\right)$, where $t_{0}=0$ in Case 1 and $t_{0}=-\infty$ in Case 2. Moreover, if there 
were a $t_{1} \in\left(t_{0},+\infty\right)$ with $\dot{g}\left(t_{1}\right) \geq 0$ we would have $\lim _{t \rightarrow+\infty}|g(t)|=+\infty$, which is impossible because of (a). Therefore, $\dot{g}(t)<0$ for all $t$ and, because of (a), $g(t)>1$ for all $t \in\left(t_{0},+\infty\right)$, and

$$
\lim _{t \rightarrow t_{0}} g(t)<1 \text {. }
$$

This, together with (4), imply (i).

In order to show (iii), we make a comparison argument with the solutions of the following equation:

$$
v^{\prime \prime}(r)+\frac{N-1}{r} v^{\prime}(r)-\frac{N-1}{r^{2}} v(r)=0 .
$$

Two linear independent solutions of this equation are $v_{0}(r)=r$, and $v_{1}(r)=$ $r^{1-N}$. Now,

$$
\int_{0} v_{0}(r) v_{1}(r)\left|\frac{Z}{r}-\lambda\right| r^{N-1} d r=\int_{0} r\left|\frac{Z}{r}-\lambda\right| d r<\infty,
$$

and, therefore, from [7, Theorem 9.1, Chapter XI] it follows that there exist two solutions $h_{0}$ and $h_{1}$ of (3) such that

$$
\lim _{r \rightarrow 0} \frac{h_{i}(r)}{v_{i}(r)}=1, \quad i=0,1 .
$$

Therefore, for any solution $h$ of (3) $\lim _{r \rightarrow 0} r^{N-1} h(r)$ exists, and it is finite. This limit will be denoted by $C$ and depends on $N, Z$, and $\lambda$. Moreover, $C(N, Z, \lambda)$ is strictly positive. In fact, if $C$ were zero, we would have $\lim _{r \rightarrow 0} h(r) / r=a \in \mathbb{R}$, which in turn implies

$$
\lim _{r \rightarrow 0} \exp \left(-r \lambda^{1 / 2}\right) g(t) / r^{1+K}=a,
$$

because of (4) and the definition of $w$. From here it would follow that $\lim _{t \rightarrow t_{0}} g(t)=0$, which contradicts (7). To finish the proof of the lemma we need only show (6). Since $t^{\prime}(r)=r^{1-N} / w(r)^{2}$, we have

$$
\lim _{r \rightarrow+\infty} \frac{t^{\prime}(r)}{t(r)}=\lim _{r \rightarrow+\infty} \frac{t^{\prime \prime}(r)}{t^{\prime}(r)}=\lim _{r \rightarrow+\infty}-2 w^{\prime} / w=+2 \lambda^{1 / 2} .
$$

Hence,

$$
\begin{aligned}
\int^{+\infty} t X(t) d t & =(K+1)(N-1-K) \int^{+\infty} t(r) /\left(t^{\prime}(r) r^{2}\right) d r \\
& \sim \frac{(K+1)(N-1-K)}{2 \lambda^{1 / 2}} \int^{+\infty} d r / r^{2}<\infty .
\end{aligned}
$$

Lemma 5. Let $Z>0$ and $\lambda>Z^{2}$. Then there exists a solution of

$$
h^{\prime \prime}(r)+\frac{1}{r} h^{\prime}(r)+\frac{Z}{r} h(r)-\lambda h(r)=0, \quad r \in(0,+\infty),
$$

such that

(i) $h>0$ in $(0, \infty)$, 
(ii) $\lim _{r \rightarrow \infty} \exp \left(r \lambda^{1 / 2}\right) r^{K} h(r)=1$, where $K=\frac{1}{2}\left(1-Z \lambda^{-1 / 2}\right)$, and $\lim _{r \rightarrow \infty} h^{\prime}(r) / h(r)=-\lambda^{1 / 2}$

(iii) $\lim _{r \rightarrow 0} \frac{h(r)}{|\log (r)|}=C(Z, \lambda)$, where $C$ is a positive constant.

Proof. Let $m(r)=\left(r / \lambda^{1 / 2}\right)^{1 / 2} h\left(r / \lambda^{1 / 2}\right)$. Then $m(r)$ satisfies

$$
m^{\prime \prime}(r)=\left(1-\frac{1}{4 r^{2}}-\frac{1}{r} Z \lambda^{-1 / 2}\right) m(r), \quad r \in(0,+\infty) .
$$

It follows rom [1, Theorem 12, p. 132] that there exists a solution $m_{0}(r)$ of (9) such that

$$
\lim _{r \rightarrow \infty} \exp (r) r^{K-1 / 2} m_{0}(r)=1 .
$$

Moreover,

$$
\lim _{r \rightarrow \infty} \frac{m_{0}^{\prime}(r)}{m_{0}(r)}=-1
$$

(see [1, Remark 13, p. 127]). If we now choose

$$
h(r)=\lambda^{(2 K-1) / 4} m_{0}\left(r \lambda^{1 / 2}\right) / r^{1 / 2},
$$

it is clear that $h$ satisfies (8) and condition (ii) above. In order to show that $h$ is positive in $(0, \infty)$ let us consider the transformation

$$
h(r)=H(t) \exp \left(-\lambda^{1 / 2} r\right),
$$

with

$$
t^{\prime}(r)=\frac{d t}{d r}=\exp \left(2 \lambda^{1 / 2} r\right) / r .
$$

It follows from (8) that $H(t)$ satisfies

$$
\ddot{H}(t)=X(t) H(t), \quad t \in(-\infty, \infty),
$$

where

$$
X(t)=\frac{\lambda^{1 / 2}-Z}{\left(t^{\prime}\right)^{2} r} .
$$

Since $\lambda>Z^{2}, X(t)>0$. Therefore, any solution of (10) vanishes at most once in $(-\infty, \infty)$. Moreover, condition (ii) above implies

$$
\lim _{r \rightarrow \infty} r^{K} H(t(r))=1 \text {, }
$$

and

$$
\lim _{r \rightarrow \infty} r^{K-1} \dot{H}(t(r)) \exp \left(2 \lambda^{1 / 2} r\right)=0 .
$$

Since $K>0$, we have that $\lim _{t \rightarrow \infty} H(t)=0$, and therefore $H(t)>0$ in $(-\infty, \infty)$. Hence, $h(r)>0$ in $(0,+\infty)$. Being both $X(t)$ and $H(t)$ positive, it follows from $(10)$ that $\dot{H}(t)<0$, for all $t$. Since $H$ is convex, $\lim _{t \rightarrow-\infty} H(t)=+\infty$, and thus, $\lim _{r \rightarrow 0} h(r)=+\infty$. Now, we make a comparison argument between the solutions of $(8)$ and those of the following:

$$
g^{\prime \prime}(r)+\frac{1}{r} g^{\prime}(r)=0, \quad r \in(0,+\infty) .
$$


Proceeding as in the proof of Lemma 4, it follows from [7, Theorem 9.1, Chapter XI] that either $\lim _{r \rightarrow 0} h(r)=C$ or $\lim _{r \rightarrow 0} h(r) /|\log (r)|=C$, where $C$ denotes a positive constant. Since $\lim _{r \rightarrow 0} h(r)=+\infty$, (iii) must hold.

\section{SPHERICAL SYMMETRY OF SOlUTIONS}

Here we will prove two different results about the spherical symmetry of solutions to (1). For the first theorem (Theorem 1) to hold, we need that $a(u)-$ $\lambda u$ be nondecreasing in $u$, for $-\lambda$ smaller than the second eigenvalue of the linear part of (1). In principle, this theorem does not need the positivity of the solutions. However, we believe that for these values of $\lambda$ there are only positive solutions to (1). The proof of this theorem uses a method introduced by L. Veron [15] (see also [14, 16]). The second result (Theorem 2) holds under more stringent conditions on the nonlinearity, namely, $a(u) / u$ has to be increasing as a function of $u$. The proof of this second theorem uses the maximum principle and reflections under planes, following the ideas introduced by Gidas, Ni, and Nirenberg [5, 6].

Proof of Theorem 1. We will consider two cases separately.

Case 1. $\lambda>(Z /(N+1))^{2}$. Let

$$
\psi(r)=\left(\int_{S^{N-1}}(u(r \sigma)-\bar{u}(r))^{2} d \sigma\right)^{1 / 2}
$$

and $H=\{r>0 \mid \psi(r)>0\}$. Let $(\alpha, \beta)$ be a connected component of $H$. On $(\alpha, \beta), \psi$ satisfies the following inequality:

$$
\psi^{\prime \prime}(r)+\frac{N-1}{r} \psi^{\prime}(r)-\frac{N-1}{r^{2}} \psi+\frac{Z}{r} \psi-\lambda y \geq \frac{1}{\psi} \int_{S^{N-1}}(g(u)-g(\bar{u}))(u-\bar{u}) .
$$

This type of inequality was first considered by L. Veron [15, Lemma 2.2] (see also [14, Lemma 3.1] and [16]). Because of the hypothesis on $g$, it follows from (13) that $\psi$ satisfies

$$
\psi^{\prime \prime}(r)+\frac{N-1}{r} \psi^{\prime}(r)-\frac{N-1}{r^{2}} \psi(r)+\frac{Z}{r} \psi(r)-\lambda \psi(r) \geq 0 .
$$

Let us define $w(t)$ by $\psi(r)=h(r) w(t)$, where $h$ is the solution of

$$
h^{\prime \prime}(r)+\frac{N-1}{r} h^{\prime}(r)-\frac{N-1}{r^{2}} h(r)+\frac{Z}{r} h(r)-\lambda h(r)=0,
$$

constructed in Lemma 4, and $t=\int_{0}^{r} s^{1-N} / h(s)^{2} d s$. The function $w(t)$ just defined satisfies $\ddot{w}(t) \geq 0$ in $(\bar{\alpha}, \bar{\beta})$, where $\bar{\alpha}=t(\alpha)$ and $\bar{\beta}=t(\beta)$. Therefore, either $\alpha=0$ or $\beta=+\infty$. On the other hand,

$$
\begin{aligned}
\lim _{t \rightarrow+\infty} \frac{w(t)}{t} & =\lim _{r \rightarrow \infty} \frac{\psi(r)}{h(r) t(r)}=2 \lambda^{1 / 2} \lim _{r \rightarrow \infty} \frac{\psi(r)}{h(r) t^{\prime}} \\
& =2 \lambda^{1 / 2} \lim _{r \rightarrow \infty} r^{N-1} h(r) \psi(r)=0,
\end{aligned}
$$


and

$$
\lim _{t \rightarrow 0} w(t)=\lim _{r \rightarrow 0} \frac{\psi(r)}{h(r)}=C \lim _{r \rightarrow 0} r^{N-1} \psi(r)=0
$$

The second equality in (15) is a consequence of Lemma 4(iii). We thus have a convex function $w$ satisfying (14) and (15). The only possibility for such a function is to vanish identically in $[0,+\infty)$. Therefore $u \equiv \bar{u}$.

Case 2. $\lambda=(Z /(N+1))^{2}$ and $g(u)$ increasing. Proceeding as in the previous case, we conclude that $w(t)$ is a convex function of $t$. In this case, however, $t \in(-\infty,+\infty)$ for $r \in(0,+\infty)$, and $h(r)=r \exp (-Z r /(N+1))$. Moreover, (14) still holds in this case, and

$$
\lim _{t \rightarrow-\infty} \frac{w(t)}{t}=\lim _{r \rightarrow 0} \frac{\psi(r)}{t h(r)}=\lim _{r \rightarrow 0} \frac{\psi(r)}{t^{\prime} h(r) r} \frac{r t^{\prime}}{t} .
$$

However,

$$
\lim _{r \rightarrow 0} \frac{r t^{\prime}}{t}=\lim _{r \rightarrow 0} \frac{r t^{\prime \prime}+t^{\prime}}{t^{\prime}}=-N .
$$

Using this back, in (16), we have

$$
\lim _{t \rightarrow-\infty} \frac{w(t)}{t}=-N \lim _{r \rightarrow 0} \frac{\psi(r)}{t^{\prime} h(r) r}=-N \lim _{r \rightarrow 0} r^{N-1} \psi(r)=0 .
$$

Hence, the only possibility is to have $\dot{w}=0$, i.e. $w=C$, where $C$ is a constant. From here, it follows that $\psi(r)=C h(r)$. Going back to (13), using the equation satisfied by $h$, we conclude

$$
\frac{1}{\psi} \int_{S^{N-1}}(g(u)-g(\bar{u}))(u-\bar{u}) \leq 0,
$$

which implies $u \equiv \bar{u}$, because $g$ is increasing.

Before we prove our second result, we will need some geometric preliminaries. For $n$ a unit vector in $\mathbb{R}^{N}$ and $d \geq 0$, let $P(n, d)$ be the plane

$$
P(n, d)=\left\{x \in \mathbb{R}^{N} \mid x \cdot n=d\right\},
$$

and let $P^{+}(n, d)$ denote the open half-space

$$
P^{+}(n, d)=\left\{x \in \mathbb{R}^{N} \mid x \cdot n<d\right\},
$$

and $P^{-}(n, d)=\mathbb{R}^{N} \backslash\left(P^{+}(n, d) \cup P(n, d)\right)$. For any function $f: \mathbb{R}^{N} \rightarrow \mathbb{R}$, we define $f^{+}(x)=f(x)$,

$$
f^{-}(x)=f(x+2(d-x \cdot n) n)
$$

for every $x \in P^{+}(n, d) \cup P(n, d)$, i.e. $f^{-}$is just the reflection of $f$ through the plane $P(n, d)$. First we prove the following theorem. 
Theorem 6. Assume that $a(s) / s$ is nondecreasing in $(0, \infty)$ and let $u$ be a positive solution of

$$
-\Delta u-Z u /|x|+a(u)=0 \quad \text { in } \mathbb{R}^{N} \quad(N \geq 2)
$$

such that $u(x) \rightarrow 0$ as $|x| \rightarrow \infty$. Let $n \in \mathbb{R}^{N}$ be a unit vector, and $d \geq 0$. Then $u^{+}(x) \geq u^{-}(x)$ for all $x \in P^{+}(n, d)$.

Remark. The idea of the proof of this theorem is taken from the results of Gidas, $\mathrm{Ni}$, and Nirenberg [5, 6]. See also [2].

Proof. Let $V(x)=Z /|x|$. It is clear that $V^{+}(x)>V^{-}(x)$, for all $x \in P^{+}(n, d)$ (see e.g. [2, Lemma 5]). Since $a(u)$ is locally Lipschitz continuous for $u \geq 0$, and $a(0)=0$, it follows that any positive solution of $(1)$ is continuous in $\mathbb{R}^{N}$ (see, e.g. [4, Lemma 6]). Let $w(x)=u^{+}(x)-u^{-}(x)$. Certainly, $w(x)=0$ for $x \in \partial P^{+}(n, d)=P(n, d)$ and at infinity. Let $D=\left\{x \in P^{+} \mid w(x)<0\right\}$. Since $u$ is continuous $D$ is open. On $D,-V^{+}(x)+a\left(u^{+}\right) / u^{+} \leq-V^{-}(x)+a\left(u^{-}\right) / u^{-}$. Therefore, from [8, Theorem 2.1], it follows that $D$ is empty and the theorem follows.

Remark. In using [8, Theorem 2.1] we need $V \in L^{1}(D)$, which restricts us to have $N \geq 2$.

The proof of theorem 2 is an immediate consequence of Theorem 6:

Proof of Theorem 2. This follows from Theorem 4, letting $d \rightarrow 0$ and noting that $n$ is an arbitrary unit vector in $\mathbb{R}^{N}$.

\section{NONEXISTENCE RESULTS}

Analogous methods to those used in the previous section to show the symmetry properties of the solutions of (1), can be used to show the nonexistence of nontrivial solutions to the same equation, for certain types of nonlinearities. Again, we impose some decay properties on the properties on the spherical average of the solution both at the origin and at infinity. For technical reasons we consider separately the cases $N=2$ and $N \geq 3$. We begin with

Proof of Theorem $3(N \geq 3)$. From Kato's inequality $[9,13]$ and $(2)$, we have

$$
\Delta|u|+Z|u| /|x|-\lambda|u| \geq(a(u)-\lambda u) \operatorname{sgn}(u) .
$$

Let $y(r)=\int_{S^{N-1}}|u(r \sigma)| d \sigma$ be the spherical average of $u$. Inequality (17) and $(a(u)-\lambda u) \operatorname{sgn}(u) \geq 0$ imply

$$
y^{\prime \prime}(r)+\frac{N-1}{r} y^{\prime}(r)+\frac{Z}{r} y(r)-\lambda y(r) \geq 0, \quad 0<r<\infty .
$$

As in the proof of Lemma 4, define $v(t)$ by $y(r)=v(t) w(r)$, where $w(r)=$ $\exp \left(-r \lambda^{1 / 2}\right) / r^{K}$ and $t^{\prime}(r)=d t / d r=r^{1-N} / w^{2}$. Then, $v(t)$ satisfies

$$
\ddot{v}(t) \geq \frac{K(N-2-K)}{\left(r t^{\prime}\right)^{2}} v(t) .
$$


Since $\lambda>(Z /(N-1))^{2}, K>0$. Also, from the definition of $K$, and $N \geq 3$, it follows that $-K+N-2>0$. Therefore $v(t)$ is convex. Moreover,

$$
\begin{aligned}
\lim _{t \rightarrow+\infty} \frac{v(t)}{t} & =\lim _{r \rightarrow+\infty} \frac{y(r)}{w(r) t}=\lim _{r \rightarrow+\infty} \frac{y(r)}{w(r) t^{\prime}(r)} \lim _{r \rightarrow+\infty} \frac{t^{\prime}(r)}{t(r)} \\
& =2 \lambda^{1 / 2} \lim _{r \rightarrow+\infty} \frac{y(r)}{r^{1-N} w^{-1}}=0,
\end{aligned}
$$

where the last inequality follows from the definition of $y$ and $w$, and hypothesis (ii). Now, let $H(t)$ be the solution of

$$
\ddot{H}(t)=\frac{K(N-2-K)}{\left(r t^{\prime}\right)^{2}} H(t)
$$

such that $\lim _{t \rightarrow+\infty} H(t)=1\left(\right.$ and $\left.\lim _{t \rightarrow+\infty} t \dot{H}(t)=0\right)$, and

$$
\lim _{r \rightarrow 0} r^{N-2-K} H(t(r))=C>0 .
$$

The construction of $H(t)$ is analogous to the construction of the function $h$ of Lemma 4, except that now we consider the equation $h^{\prime \prime}(r)+((N-1) / r) h^{\prime}(r)=$ 0 to study the behavior of $H(t(r))$ at $r=0$.

Let $G(t) \equiv \dot{v} H-\dot{H} v$ be the wronskian between $v$ and $H$. Then, $\dot{G} \geq 0$ and therefore $G(t)$ is nondecreasing. Since $\lim _{t \rightarrow+\infty} G(t)=0$, we get

$$
\frac{v(t)}{v(s)} \leq \frac{H(t)}{H(s)} \text { for } t \geq s .
$$

Hypothesis (i), the definition of $v$, and the properties of $H$ imply

$$
\lim _{r \rightarrow 0} \frac{v(s(r))}{H(s(r))}=0
$$

which, combined with (18), in turn implies $v(t) \equiv 0$ and, therefore, $u \equiv 0$.

Proof of Theorem $3(N=2)$. Let $y(r)=\int_{S^{1}}|u(r \sigma)| d \sigma$ be the spherical average of $|u|$. As in the case $N \geq 3,(a(u)-\lambda u) \operatorname{sgn}(u) \geq 0$ and Kato's inequality imply

$$
y^{\prime \prime}(r)+\frac{1}{r} y^{\prime}(r)+\frac{Z}{r} y(r)-\lambda y(r) \geq 0, \quad 0<r<\infty .
$$

Define $v(t)$ by $y(r)=v(t) \exp \left(-r \lambda^{1 / 2}\right)$, where $t^{\prime}(r)=\exp \left(2 r \lambda^{1 / 2}\right) / r$. Then, $v$ satisfies

$$
\ddot{v}(t) \geq X(t) v(t), \quad t \in(-\infty, \infty)
$$

in the sense of distributions, where

$$
X(t)=\frac{\lambda^{1 / 2}-Z}{\left(t^{\prime}\right)^{2} r}>0 .
$$

Now, let $h(r)$ and $H(t)$ be defined as in Lemma 5 above. Then, $H$ satisfies $\ddot{H}(t)=X(t) H(t)$. Define $G(t)=\dot{v}(t) H(t)-v(t) \dot{H}(t)$. Hence, $\dot{G}(t) \geq 0$, for 
all $t \in(-\infty, \infty)$. Moreover, $G(t)=\dot{v} r^{-K}(1+o(1))-v r^{1-K} \exp \left(-2 \lambda^{1 / 2} r\right) o(1)$ for $t \rightarrow \infty$ and, by L'Hôpital,

$$
\lim _{r \rightarrow+\infty} \frac{\dot{v}}{r^{K}}=\lim _{r \rightarrow+\infty} \frac{v}{r^{K} t(r)}=2 \lambda^{1 / 2} \lim _{r \rightarrow+\infty} r^{1-K} \exp \left(\lambda^{1 / 2} r\right) y(r)=0 .
$$

Hence, $\lim _{t \rightarrow+\infty} G(t)=0$, and therefore,

$$
v(t) / v(s) \leq H(t) / H(s) \text { for } t \geq s .
$$

Hypothesis $\left(\mathrm{i}^{\prime}\right)$, the definition of $v$, and the properties of $H$ imply

$$
\lim _{r \rightarrow 0} \frac{v(s(r))}{H(s(r))}=0
$$

which, combined with (19), in turn implies $v(t) \equiv 0$ and, therefore, $u \equiv 0$.

One can extend Theorem 3 to include the case $\lambda=(Z /(N-1))^{2}$. We have Proposition 7. Let $\lambda=(Z /(N-1))^{2}, Z>0$, and $(a(u)-\lambda u) \operatorname{sgn}(u)>0$ if $|u|>0$. Then, if $u$ is a solution of (2) (in the sense of distributions) such that

(i) $\lim _{r \rightarrow 0} r^{N-2} \int_{S^{N-1}}|u(r \sigma)| d \sigma=0$ for $N \geq 3$, or

(i') $\lim _{r \rightarrow 0}|\log (r)|^{-1} \int_{S^{1}}|u(r \sigma)| d \sigma=0$ for $N=2$, and

(ii) $\lim _{r \rightarrow \infty} \exp \left(-r \lambda^{1 / 2}\right) r^{-K+N-1} \int_{S^{N-1}}|u(r \sigma)| d \sigma=0$, for $N \geq 2$ with $K=$ $\frac{1}{2}\left((N-1)-Z \lambda^{-1 / 2}\right)$,

we have $u \equiv 0$.

Proof. We proceed analogously as we did in the proof of Theorem 3. However, in this case, $\lim _{t \rightarrow \pm \infty} v(t) / t=0$. this, together with $v$ being convex implies $\dot{v} \equiv 0$, hence $v$ is a constant, and therefore $y=c w$. From here it follows that

$$
0=y^{\prime \prime}(r)+\frac{N-1}{r} y^{\prime}(r)+\frac{Z}{r} y(r)-\lambda y(r) \geq \int_{S^{N-1}}(a(u)-\lambda u) \operatorname{sgn}(u) d \sigma,
$$

and therefore $u \equiv 0$.

Remark. Theorem 3 and Proposition 7 still hold if we replace $a(u)$ by $a(u)+$ $\int u(y)^{2}|x-y|^{-1} d y$ in (2) (i.e. if we include the electronic repulsion).

Proof. After completion of this work, we received a preprint by L. Veron (Geometric invariance of singular solutions of some nonlinear partial differential equations) where he considers some problems related to our Theorem 1. While our method is similar to the one used by Veron, the class of nonlinearities we handle, contains the class considered by Veron for (1). Specifically, we allow $\lambda \geq(Z /(N+1))^{2}$, while he only allows $\lambda \geq(Z / N)^{2}$.

\section{REFERENCES}

1. R. Bellman, Stability theory of differential equations, New York, Dover, 1953.

2. R. Benguria, Dependence of the Thomas-Fermi energy on the nuclear coordinates, Comm. Math. Phys. 81 (1981), 419-428. 
3. R. Benguria, H. Brezis, and E. H. Lieb, The Thomas-Fermi-von Weizsäcker theory of atoms and molecules, Comm. Math. Phys. 79 (1981), 167-180.

4. R. Benguria and L. Jeanneret, Existence and uniqueness of positive solutions of semilinear elliptic equations with Coulomb potentials on $\mathbb{R}^{3}$, Comm. Math. Phys. 104 (1986), 291-306.

5. B. Gidas, W.-M. Ni, and L. Nirenberg, Symmetry and related properties via the maximum principle, Comm. Math. Phys. 68 (1979), 209-243.

6. $\ldots$, Symmetry of positive solutions of nonlinear equations in $\mathbb{R}^{N}$, Math. Anal. Appl. 7A (1981), 369-402.

7. P. Hartman, Ordinary differential equations, Wiley, New York, 1964.

8. T. Hoffmann-Ostenhof, A comparison theorem for differential inequalities with applications in quantum mechanics, J. Phys. A 13 (1980), 417-424.

9. T. Kato, Schrödinger operators with singular potentials, Israel J. Math. 13 (1973), 135-148.

10. E. H. Lieb, Thomas-Fermi and related theories of atoms and molecules, Rev. Modern Phys. 53 (1981), 603-641.

11. __ Analysis of the Thomas-Fermi-von Weizsäcker equation for an infinite atom without electron repulsion, Comm. Math. Phys. 85 (1982), 15-25.

12. E. H. Lieb and D. A. Liberman, Numerical calculation of the Thomas-Fermi-von Weizsäcker function for an infinite atom without electron repulsion, Los Alamos Report No. LA-9186MS, 1982.

13. M. Reed and B. Simon, Methods of modern mathematical physics. II: Fourier analysis, self-adjointness, Academic Press, New York, 1975.

14. J. L. Vazquez and C. Yarur, Isolated singularities of the solution of the Schrödinger equation with a radial potential, Arch. Rational Mech. Anal. 98 (1987), 251-284.

15. L. Veron, Singular solutions of some nonlinear elliptic equations, Nonlinear Anal. 5 (1981), 225-242.

16. C. Yarur, Singularidades de ecuaciones de Schrödinger estacionarias, Tesis de Doctorado, Universidad Complutense de Madrid, 1984.

Departamento de Fisica, F.C.F.M., Universidad de Chile, Casilla 487-3, Santiago, Chile (Bitnet address: RBENGURI AT UCHCECVM)

Departamento de Matemáticas, Universidad de Santiago de Chile, Casilla 5659, Correo 2, Santiago, Chile (Bitnet address: CYARUR at USACHVM1) 